\title{
PYRIMIDINE INCORPORATED SCHIFF BASE OF ISONIAZID WITH THEIR SYNTHESIS, CHARACTERIZATION AND IN VITRO BIOLOGICAL EVALUATION
}

\author{
HETAL I SONI, NAVIN B PATEL* \\ Department of Chemistry, Veer Narmad South Gujarat University, Surat, Gujarat, India. Email: drnavinbpatel@gmail.com
}

Received: 20 April 2017, Revised and Accepted: 24 June 2017

\begin{abstract}
Objective: Versatile biological activities of nitrogen containing heterocycles in medicinal chemistry, mainly pyrimidine and pyridine ring based heterocyclic moieties are very important. Pharmaceutical important of pyrimidine and isoniazid moiety prompted us to synthesize isoniazid clubbed pyrimidine derivatives and evaluated for antimicrobial and antituberculosis activity.

Method: 2-(2-(3-bromo benzylidene)-1-isonicotinoyl hydrazinyl)- $N$-(4-(substituted phenyl)-6-(substituted aryl) pyrimidin-2-yl) acetamide 2 (A-) have been synthesized by condensation reaction of 2-chloro- $N$-[4-(substituted phenyl)-6-(substituted aryl) pyrimidin-2-yl] acetamide and $N$ - $[(E)$ (3-bromophenyl) methylidene]pyridine-4-carbohydrazide. All newly synthesized compounds were screened for in vitro antibacterial activity against Escherichia coli, Pseudomonas aeruginosa, Staphylococcus aureus, and Staphylococcus pyogenes, antifungal against Candida albicans, Aspergillus niger, and Aspergillus clavatus, and antituberculosis activity against Mycobacterium tuberculosis $\mathrm{H}_{37} \mathrm{RV}$.
\end{abstract}

Results: Majority of the compounds exhibited good antibacterial, antifungal, and antituberculosis activity. All titled compounds were characterized by spectral analyses (infrared spectroscopy, nuclear magnetic resonance spectroscopy, and mass spectroscopy).

Conclusion: 2-(2-(3-bromo benzylidene)-1-isonicotinoyl hydrazinyl)- $N$-(4-(substituted phenyl)-6-(substituted aryl) pyrimidin-2-yl) acetamide 2 (A-) showed good antimicrobial activity and comparatively good antituberculosis activity. Hence, all the compounds of this series considered for future investigation mainly in area of antibacterial, antifungal study.

Keywords: Pyrimidine, Pyridine, Isoniazid, Antimicrobial, Antituberculosis.

(C) 2017 The Authors. Published by Innovare Academic Sciences Pvt Ltd. This is an open access article under the CC BY license (http://creativecommons. org/licenses/by/4. 0/) DOI: http://dx.doi.org/10.22159/ajpcr.2017.v10i10.19302

\section{INTRODUCTION}

Isoniazid (isonicotinic acid hydrazide) used as the first-line antibiotic for tuberculosis treatment since 1952. It is prodrug which is activated by Mycobacterium tuberculosis catalase-peroxidase (Kat G) is a bifunctional hemoprotein [1]. The primary target of isoniazid (INH) is the enoyl-ACP reductase enzyme (InhA) from $M$. tuberculosis. Isoniazid is a derivative of nicotinic acid, which possesses antibacterial, antioxidant, antiinflammatory, and anticarcinogenic activities and showed putative activity against osteoarthritis and granuloma annulare. Nicotinic acid (Niacin or vitamin $\mathrm{B}_{3}$ ) derivatives are also very important starting material to prepare bioactive moieties [2]. Likewise, pyrimidine also appeared as an important pharmacophore used as building blocks of numerous natural compounds and found in vitamins (thiamine, riboflavin, and folic acid), lipopolysaccharides, DNA, and RNA. The synthetic drugs such as HIV drugs, barbiturates, anticancer agents, antimicrobial, antiviral, antimalarial, and anti-inflammatory [3-9]. Structural modification of pyrimidine with different functional groups or other heterocycles may improve clinical outcomes [10-19]. Due to this importance of isoniazid (nicotinic acid derivatives) and pyrimidine nucleus, we thought to synthesize isoniazid clubbed pyrimidine derivatives and evaluated in vitro antibacterial activity against several kinds of bacteria, fungi, and M. tuberculosis $\mathrm{H}_{37} \mathrm{Rv}$.

In this study, newly synthesized 2-(2-(3-bromo benzylidene) -1-isonicotinoyl hydrazinyl) $-N$-(4-(substituted phenyl) -6-(substituted aryl) pyrimidin-2-yl) acetamide $2_{(A-I)}$ were identified and characterized by spectral analysis (IR, nuclear magnetic resonance [NMR], and mass) and elemental analysis. Antibacterial studies were performed toward Escherichia coli, Pseudomonas aeruginosa, Staphylococcus aureus and Staphylococcus pyogenes, antifungal activities against Candida albicans, Aspergillus niger, and Aspergillus clavatus. The majority of the compounds were showed a high degree of evaluation toward Gram-positive and Gram-negative bacteria as compared to standard drug ampicillin. Antifungal activity of many moieties was comparatively high as compared with standard drug Griseofulvin. Where some of the newly synthesized compounds showed good antitubercular activity.

\section{METHODS}

The chemicals used in the synthesis were purchased from commercial sources were of analytical grade and were used without further purification. Laboratory Chemicals were supplied by Sigma-Aldrich and Fisher Scientific Ltd. Melting points were determined by the open tube capillary method and are uncorrected. The purity of the compounds was determined by thin layer chromatography (TLC) plates (silica gel G) using different eluent system. The IR spectra were obtained on Fourier transform infrared spectroscopy (FT-IR). Infrared spectrophotometer model RZX (Perkin Elmer) and Agilent resolution Pro FT-IR spectrometer using potassium bromide pellets; the frequencies are expressed in $\mathrm{cm}^{-1}$. The ${ }^{1} \mathrm{H}-\mathrm{NMR}$ and ${ }^{13} \mathrm{C}$-NMR spectra were recorded on a Bruker Advance II 400 spectrometer (100 MHz FT-NMR) using tetramethylsilane as the internal standard in deuterochloroform $\left(\mathrm{CDCl}_{3}\right)$ and dimethyl sulfoxide (DMSO-d ) (chemical shifts in $\delta \mathrm{ppm}$ ). Mass spectra recorded WATERS Q-T of micro mass (electrospray ionization-MS). Column chromatography was performed on silica gel 60 (0.043-0.06 mm) Merck. Elemental analysis was performed on Carlo Erba1108 analyzer, and the result was varying within $\pm 0.04 \%$ of the calculated values.

\section{General method of synthesis}

Synthesis of (2E)-1-(4-substituted phenyl)-3-(substituted phenyl) prop-2-en-1-one derivatives (A)

$\alpha-\beta$ unsaturated ketone [20] compounds were prepared by reported method [21-24]. 
Synthesis of 4-(4-substituted phenyl)-6-(substituted phenyl) pyrimidin-2-amine derivatives $(B)$

A synthesized compound (A) $(10 \mathrm{mmol})$, guanidine nitrate $(15 \mathrm{mmol})$ and were dissolved in ethanol $(10 \mathrm{~mL})$ and added sodium methoxide in methanol $(25 \%, 20 \mathrm{~mL})$ was refluxed for $6-8 \mathrm{hrs}$. The progress of the reaction was monitored by TLC using toluene:ethyl acetate (2.5:7.5), interval of every 30 minutes and after completion of the reaction, the mixture was cooled, diluted with water and filtered. The separated solid compound was washed with water, dried and recrystallized with ethanol to get (B).

Synthesis of 2-chloro-N-[4-(substituted phenyl)-6-(4-substituted phenyl)pyrimidin-2-yl] acetamide (C)

A synthesized compound (B) (10 mmol) dissolved in dichloromethane $(15 \mathrm{~mL})$ and dropwise addition of chloroacetyl chloride $(15 \mathrm{mmol})$. Then added triethylamine $(10 \mathrm{mmol})$. Reaction mass was stirred for $3 \mathrm{hrs}$. The progress of the reaction was monitored by TLC using toluene: methanol:ethyl acetate (2:3:5), interval of every 30 minutes and after completion of the reaction, the mixture diluted with water and the organic layer was separated. The separated liquid compound was dried, washed water and recrystallized with ethanol to get (C).

Synthesis of $N^{\prime}-[(E)-(3-b r o m o p h e n y l)$ methylidene]pyridine-4carbohydrazide (D)

This compound was prepared by reported method $[25,26]$.

Synthesis of 2-(2-(3-Bromo benzylidene)-1-isonicotinoyl hydrazinyl)-N-(4-(substituted phenyl)-6-(substituted aryl) pyrimidin-2-yl) acetamide (E)

A synthesized compound (D) (10 mmol) was dissolved in $15 \mathrm{~mL}$ methanol, and $3.4 \mathrm{~g}$ of $\mathrm{K}_{2} \mathrm{CO}_{3}$ was added. The reaction mixture was refluxed on a water bath. A dropping funnel was fitted to the round bottom flask, and in the dropping funnel, a solution of synthesized compound (C) $(10 \mathrm{mmol})$ in $20 \mathrm{~mL}$ methanol was taken. A slow addition of this solution was done. The reaction mixture was refluxed in water bath at $80^{\circ} \mathrm{C}$ for $4 \mathrm{hrs}$. The reaction was monitored by TLC using toluene:ethyl acetate (2:8). After completion of the reaction, the mixture was kept at room temperature. After filtration, it was washed with water and crystallized from ethanol to get (E). The crude solid was purified by column chromatography.

\section{Biological evaluation}

In vitro biological evaluation

Minimum inhibition concentration method for antimicrobial activity.

For nutrient medium Mueller-Hinton broth was used as to grow and dilute the drug suspension for the test. Size of inoculum for test strain was adjusted to $10^{8}$ colony forming unit. DMSO was used for negative control and as diluents to get the desired concentration of drugs to test on standard bacterial strains. For primary and secondary screening serial dilutions were prepared. The control tube containing no antibiotic was immediately subcultured (before inoculation) by spreading a loopful evenly over a quarter of plate of medium suitable for the growth of the test organism and put for incubation at $37^{\circ} \mathrm{C}$ $24 \mathrm{hrs}$. The minimum inhibitory concentration (MIC) of the control organism was read to check the accuracy of the drug concentrations. The standard drug used in this method was ampicillin, chloramphenicol, ciprofloxacin, griseofulvin, and nystatin. The lowest concentration inhibiting the growth of the organism was recorded as the MIC. The amount of growth of the control tube before incubation (which represents the original inoculum) was compared. The test included a second set of the same dilutions inoculated with an organism of known sensitivity. Each synthesized drug was diluted obtaining $1000 \mu \mathrm{g} / \mathrm{mL}$ concentration, as a stock solution. In primary screening 500,250 , and $125 \mu \mathrm{g} / \mathrm{mL}$ concentrations of the synthesized drugs were taken. The active synthesized drugs found in this primary screening were further tested in a second set of dilution against all microorganisms. The drugs found active in primary screening were similarly diluted to obtain $100,50,25,12.5,6.250,3.125$, and $1.5625 \mu \mathrm{g} / \mathrm{mL}$ concentrations. The highest dilution showing at least $99 \%$ inhibition is taken as MIC (Table 1) [27,28].

\section{RESULTS}

Synthetic route for title compounds given in Figs. 1 and 2. The pyrimidine ring system was prepared by 4-(4-substituted phenyl) -6-(substituted aryl) pyrimidine-2-amine. This compound was key intermediate required for the synthesis of title compounds. This compound further reacted with chloroacetyl chloride and given 2-chloro- $N$-[4-(substituted phenyl)-6-(4- substituted aryl) pyrimidin2-yl] acetamide (A). To prepare final yield, compound (A) condensed with $N^{\prime}$-[(E)-(3-bromophenyl)methylidene]pyridine-4-carbohydrazide in the presence of $\mathrm{K}_{2} \mathrm{CO}_{3}$, Which yielded (E)-2-(2-(3-Bromo benzylidene)-1-isonicotinoyl hydrazinyl)- $N$-(4-(substituted phenyl)6-(substituted aryl) pyrimidin-2-yl) acetamide $\left(2_{\text {A-l }}\right.$ ). Spectral studies of these compounds proved the structure of derivatives. IR absorption band of compound $2_{\mathrm{A}^{\prime}}$ IR $(\mathrm{KBr}) \mathrm{cm}^{-1}: 3432.80(\mathrm{~N}-\mathrm{H}), 3190.58,3051.74$ for aromatic C-H, $1652.88(-\mathrm{CONH}), 1356.10,1252.29,1218.37$, 1171.87, $1140.44(\mathrm{C}-\mathrm{N})$, and $573.76(\mathrm{C}-\mathrm{Br}) .{ }^{1} \mathrm{H}$ NMR spectrum of the compound recorded in $\mathrm{CDCl}_{3}$, singlet of $1 \mathrm{H}$ at 8.62 for $\mathrm{CH}$ of pyrimidine ring, $17 \mathrm{H}$ of aromatic $\mathrm{CH}$ appeared at 7.31-8.77, singlet of $1 \mathrm{H}$ at 5.33 for $\mathrm{NH}$, disappeared on D2O exchange and singlet of $2 \mathrm{H}$ for $\mathrm{CH}_{2}$ recorded at $3.85 .{ }^{13} \mathrm{C}$ NMR of the final compound showed at $168.05,167.78$ for $\mathrm{C}=0,164.38$ for $\mathrm{C}=\mathrm{N}$ of pyrimidine, $155.3-113.43$ for aromatic carbon, and 54.41 for $\mathrm{CH}_{2}$ confirming the formation of a final compound.

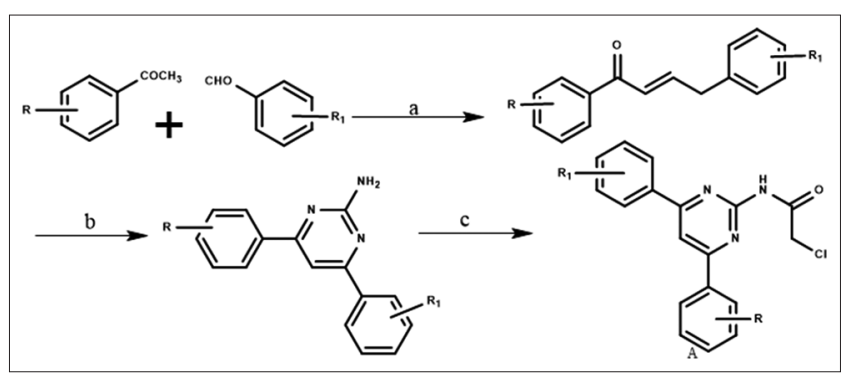

Fig. 1: General synthesis of 2-chloro-N-[4-(substituted phenyl)-6-(4- substituted phenyl) pyrimidin-2-yl] acetamide derivatives. Reagents and conditions: (a) $\mathrm{CH}_{3} \mathrm{OH}, \mathrm{NaOH}$, stirred at room temperature $2 \mathrm{hrs}$, (b) guanidine nitrate, $\mathrm{CH}_{3} \mathrm{ONa}, \mathrm{CH}_{3} \mathrm{OH}$, reflux 8 hrs (C) dichloromethane, chloroacetyl chloride, and triethylamine

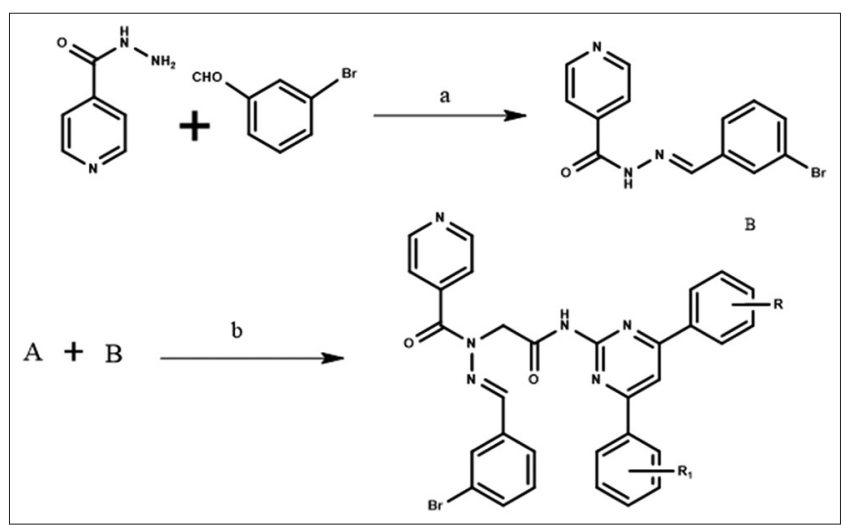

Fig. 2: General synthesis of 2-(2-(3-bromo benzylidene)1-isonicotinoyl hydrazinyl)-N-(4-(substituted phenyl)-6(substituted aryl) pyrimidin-2-yl) acetamide derivatives. Reagents and conditions: (a) $\mathrm{CH}_{3} \mathrm{OH}, \mathrm{CH}_{3} \mathrm{COOH}$, reflux $2 \mathrm{hrs}$ (b) $\mathrm{CH}_{3} \mathrm{OH}, \mathrm{K}_{2} \mathrm{CO}_{3}$ reflux $4 \mathrm{hrs}$ 
Table 1: Physical data of the compounds $\left(2_{A-j}\right)$

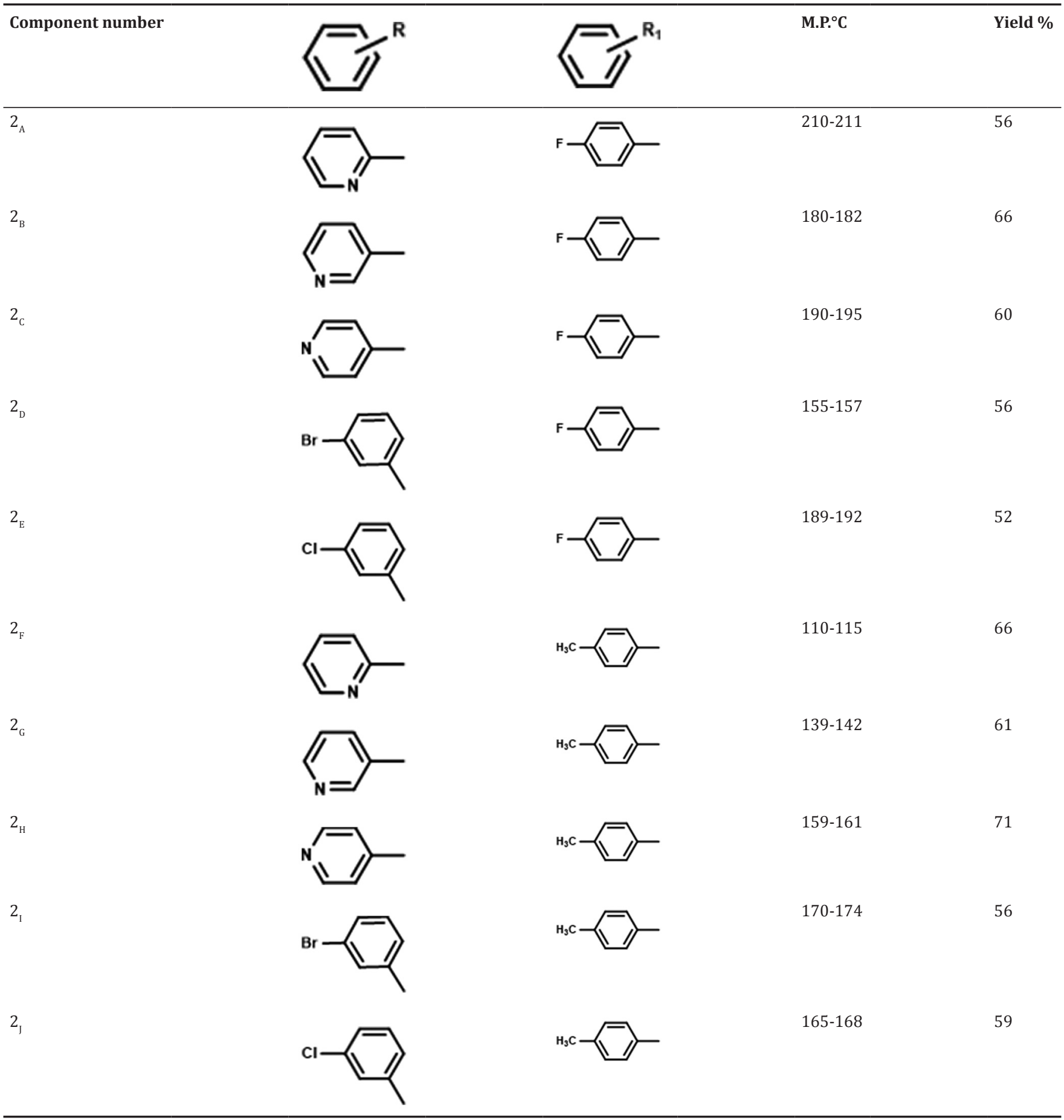

Mass spectra of the synthesized compounds showed $M+/ M+1$ peak, confirmed their molecular formula. The analytical data of synthesized compounds are as follows.

(E)-2-(2-(3-bromobenzylidene)-1-isonicotinoylhydrazinyl)- $\mathrm{N}$-(4(4-fluorophenyl)-6-(pyridin-2-yl)pyrimidin-2-yl)acetamide $2_{A}$ IR: $3432.80(\mathrm{~N}-\mathrm{H}), 3190.58,3051.74$, (C-H aromatic), 1652.88 (-CONH), 1544.72 ( $\mathrm{N}-\mathrm{H}$ bending), 1429.32, 1476.17 ( $\mathrm{C}=\mathrm{C}$ aromatic), 1356.10, 1252.29, 1218.37, 1171.87, 1140.44 (C-N), 1063.01 (C-F) 573.76 (mono substituted $\mathrm{Br}$ ). ${ }^{1} \mathrm{H}-\mathrm{NMR}\left(\mathrm{CDCl}_{3}, 400 \mathrm{MHz}\right): 8.62(\mathrm{~s}, 1 \mathrm{H}, \mathrm{CH}$ of pyrimidine ring); 7.31-8.77 ( $m, 16 \mathrm{H}, \mathrm{Ar}-\mathrm{H}) ; 8.39(\mathrm{~s}, 1 \mathrm{H}, \mathrm{CH}) ; 5.33(\mathrm{~s}, 1 \mathrm{H}$, CONH); 3.85 (s, 2H, CH2). ${ }^{13} \mathrm{C}-\mathrm{NMR}\left(\mathrm{CDCl}_{3}, 400 \mathrm{MHz}\right): 168.51,165.62$
(C=0); 162.91 ( $\mathrm{C}=\mathrm{N}, \mathrm{C}$, pyrimidine); $155.30 ; 101.30-149.71 ; 146.91$ (aromatic ring), $55.40\left(\mathrm{CH}_{2}\right)$. MS $(\mathrm{m} / \mathrm{z}): 609.09\left([\mathrm{M}+\mathrm{H}]{ }^{+}\right), 611.09$ $\left[\mathrm{M}^{+}+2\right]$, elemental analysis calculated for $\mathrm{C}_{30} \mathrm{H}_{21} \mathrm{Br} \mathrm{FN}_{7} \mathrm{O}_{2}: \mathrm{C} 59.03, \mathrm{H}$ 3.47, N 16.06; found: C 59.04, H 3.45, N 16.04.

(E)-2-(2-(3-bromobenzylidene)-1-isonicotinoylhydrazinyl)- $\mathrm{N}$-(4(4-fluorophenyl)-6-(pyridin-3-yl)pyrimidin-2-yl)acetamide $2_{B}$ IR: $3430.70(\mathrm{~N}-\mathrm{H}), 3190.58,3041.44,(\mathrm{C}-\mathrm{H}$ aromatic), 1657.18 (-CONH), 1544.72 (N-H bending), 1429.32, 1476.17 ( $\mathrm{C}=\mathrm{C}$, aromatic), 1356.10, 1252.29, 1218.37, and 1170.87 (C-N), 1061.31 (C-F) 577.66 (mono substituted $\mathrm{Br}) .{ }^{1} \mathrm{H}-\mathrm{NMR}\left(\mathrm{CDCl}_{3}, 400 \mathrm{MHz}\right): 8.66(s, 1 \mathrm{H}, \mathrm{CH}$ of pyrimidine ring); 6.68-8.78 (m, 16H, Ar-H); $8.45(s, 1 \mathrm{H}, \mathrm{CH}) ; 5.63(s, 1 \mathrm{H}, \mathrm{CONH})$; 
$3.85\left(s, 2 \mathrm{H}, \mathrm{CH}_{2}\right) \cdot{ }^{13} \mathrm{C}-\mathrm{NMR}\left(\mathrm{CDCl}_{3}, 100 \mathrm{MHz}\right): 166.24,165.74(\mathrm{C}=0)$; 160.20 (Pyrimidine); 149.97-101.75 (aromatic ring); $55.04\left(\mathrm{CH}_{2}\right)$. MS $(\mathrm{m} / \mathrm{z}): 609.09\left([\mathrm{M}+\mathrm{H}]^{+}\right), 611.09\left[\mathrm{M}^{+}+2\right]$, elemental analysis calculated for $\mathrm{C}_{30} \mathrm{H}_{21} \mathrm{Br} \mathrm{FN}_{7} \mathrm{O}_{2}$ : C 59.03, H 3.47, N 16.06; found: C 59.05, H 3.40, N 16.04 .

(E)-2-(2-(3-bromobenzylidene)-1-isonicotinoylhydrazinyl)- $N$-(4(4-fluorophenyl)-6-(pyridin -4-yl)pyrimidin-2-yl)acetamide $2_{C}$ IR: 3423.46, 3351.91 (N-H), 3125.58, 3057.84, (C-H aromatic), 1657.98 (-CONH), 1549.72 ( $\mathrm{N}-\mathrm{H}$ bending), 1429.32, 1476.87 (C=C, aromatic), 1356.10, 1252.29, 1218.37, and 1180.87 (C-N), 1067.31 (C-F) 567.86 (mono substituted $\mathrm{Br}$ ). ${ }^{1} \mathrm{H}-\mathrm{NMR}\left(\mathrm{CDCl}_{3}, 400 \mathrm{MHz}\right): 8.61(\mathrm{~s}, 1 \mathrm{H}, \mathrm{CH}$ of pyrimidine ring); 7.31-8.77 $(\mathrm{m}, 16 \mathrm{H}, \mathrm{Ar}-\mathrm{H}) ; 8.39(\mathrm{~s}, 1 \mathrm{H}, \mathrm{CH}) ; 5.33(\mathrm{~s}, 1 \mathrm{H}$, $\mathrm{CONH}) ; 3.85\left(s, 2 \mathrm{H}, \mathrm{CH}_{2}\right) \cdot{ }^{13} \mathrm{C}-\mathrm{NMR}\left(\mathrm{CDCl}_{3}, 100 \mathrm{MHz}\right): 168.51,162.91$ $(\mathrm{C}=0) ; 160.60$ (pyrimidine); 106.20-148.82 (aromatic ring); 55.41 $\left(\mathrm{CH}_{2}\right)$. MS (m/z): $609.09\left([\mathrm{M}+\mathrm{H}]^{+}\right), 611.09\left[\mathrm{M}^{+}+2\right]$, elemental analysis calculated for $\mathrm{C}_{30} \mathrm{H}_{21} \mathrm{Br} \mathrm{FN}_{7} \mathrm{O}_{2}$ : C 59.03, H 3.47, N 16.06; found: C 59.15, H 3.39, N 16.10 .

(E)-2-(2-(3-bromobenzylidene)-1-isonicotinoylhydrazinyl)$\mathrm{N}$-(4-(3-bromophenyl)-6-(4-fluorophenyl) pyrimidin-2-yl) acetamide $2_{D}$

IR: 3472.56, 3303.45 (N-H), 3056.70, (C-H aromatic), 1651.75 (-CONH), 1591.78 ( $\mathrm{N}-\mathrm{H}$ bending), 1428.37, 1472.37 (C=C, aromatic), 1308.26, 1234.07, 1165.55, and 1140.44 (C-N), 1024.41 (C-F) 573.76 (mono substituted $\mathrm{Br}) .{ }^{1} \mathrm{H}-\mathrm{NMR}\left(\mathrm{CDCl}_{3}, 400 \mathrm{MHz}\right): 8.61(s, 1 \mathrm{H}, \mathrm{CH}$ of pyrimidine ring); 7.31-8.77 ( $m, 16 \mathrm{H}, \mathrm{Ar}-\mathrm{H})$; $8.39(\mathrm{~s}, 1 \mathrm{H}, \mathrm{CH}) ; 5.35(s, 1 \mathrm{H}, \mathrm{CONH})$; $3.85(s, 2 \mathrm{H}, \mathrm{CH} 2) .{ }^{13} \mathrm{C}-\mathrm{NMR}\left(\mathrm{CDCl}_{3}, 100 \mathrm{MHz}\right): 168.54,162.92$ (C=0); 140.81-107.08; $55.41\left(\mathrm{CH}_{2}\right)$. MS (m/z): $688.09\left([\mathrm{M}+\mathrm{H}]{ }^{+}\right), 690.09$ $\left[\mathrm{M}^{+}+2\right]$, elemental analysis calculated for $\mathrm{C}_{31} \mathrm{H}_{21} \mathrm{Br}_{2} \mathrm{FN}_{6} \mathrm{O}_{2}$ : C 54.09, $\mathrm{H}$ 3.08, N 12.21; found: C 54.11, H 3.09, N 12.23.

(E)-2-(2-(3-bromobenzylidene)-1-isonicotinoylhydrazinyl)- $N$-(4(3-chlorophenyl)-6-(4-fluorophenyl)pyrimidin-2-yl)acetamide $2_{E}$ IR: $3341.42(\mathrm{~N}-\mathrm{H}), 3060.39,3033.38$ (C-H aromatic), 1673.22 (-CONH), 1553.21 ( $\mathrm{N}-\mathrm{H}$ bending), 1433.36, 1472.37 ( $\mathrm{C}=\mathrm{C}$, aromatic), 1267.26, 1218.37, 1183.49, and 1140.64 (C-N), 1024.41 (C-F), 688.12 (C-Cl), 573.76 (mono substituted $\mathrm{Br}$ ). ${ }^{1} \mathrm{H}-\mathrm{NMR}\left(\mathrm{CDCl}_{3}, 400 \mathrm{MHz}\right): 8.61(\mathrm{~s}, 1 \mathrm{H}$, $\mathrm{CH}$ of pyrimidine ring); 7.31-8.77 ( $m, 17 \mathrm{H}, \mathrm{Ar}-\mathrm{H}) ; 8.38(\mathrm{~s}, 1 \mathrm{H}, \mathrm{CH}) ; 5.35$ $(s, 1 \mathrm{H}, \mathrm{CONH}) ; 3.85\left(s, 2 \mathrm{H}, \mathrm{CH}_{2}\right) \cdot{ }^{13} \mathrm{C}-\mathrm{NMR}\left(\mathrm{CDCl}_{3}, 100 \mathrm{MHz}\right): 168.51$, $162.69(\mathrm{C}=0)$ ); $146.95-107.08$ (aromatic ring); $55.41\left(\mathrm{CH}_{2}\right)$. $\mathrm{MS}(\mathrm{m} / \mathrm{z})$ : $642.90\left([\mathrm{M}+\mathrm{H}]{ }^{+}\right), 643.88\left[\mathrm{M}^{+}+1\right], 644.09\left[\mathrm{M}^{+}+2\right]$, elemental analysis calculated for $\mathrm{C}_{31} \mathrm{H}_{21} \mathrm{Br} \mathrm{Cl} \mathrm{FN} \mathrm{O}_{2}$ : C 57.83, H 3.29, N 13.05; found: C 57.81, H 3.33, N 13.03 .

(E)-2-(2-(3-bromobenzylidene)-1-isonicotinoylhydrazinyl)- $N$-(4(pyridin-2-yl)-6-(p-tolyl) pyrimidin-2-yl)acetamide $2_{F}$

IR: 3352.46 (N-H), 3056.39, 3022.38 (C-H aromatic), 2941.41, 2932.4 (C-H), 1671.22 (-CONH), 1541.21 (N-H bending), 1413.36, 1423.38 $(\mathrm{C}=\mathrm{C}$, aromatic), 1256.26, 1234.37, 1123.49 (C-N), 567.76 (mono substituted $\mathrm{Br}) .{ }^{1} \mathrm{H}-\mathrm{NMR}\left(\mathrm{CDCl}_{3}, 400 \mathrm{MHz}\right): 8.64(\mathrm{~s}, 1 \mathrm{H}, \mathrm{CH}$ of pyrimidine ring), 7.42-8.77 (m, 16H, Ar-H); $8.39(s, 1 \mathrm{H}, \mathrm{CH}) ; 5.34(s, 1 \mathrm{H}, \mathrm{CONH})$; $3.85\left(s, 2 \mathrm{H}, \mathrm{CH}_{2}\right) ; 2.34\left(s, 3 \mathrm{H}, \mathrm{CH}_{3}\right) .{ }^{13} \mathrm{C}-\mathrm{NMR}\left(\mathrm{CDCl}_{3}, 100 \mathrm{MHz}\right): 168.35$, $165.46(\mathrm{C}=0)$; 162.76 (pyrimidine); 155.03; 149.71-101.39; 55.43 $\left(\mathrm{CH}_{2}\right), 21.34\left(\mathrm{CH}_{3}\right) \cdot \mathrm{MS}(\mathrm{m} / \mathrm{z}): 605.03\left([\mathrm{M}+\mathrm{H}]{ }^{+}\right), 606.13\left[\mathrm{M}^{+}+1\right], 607.12$ $\left[\mathrm{M}^{+}+2\right]$, elemental analysis calculated for $\mathrm{C}_{31} \mathrm{H}_{24} \mathrm{BrN}_{7} \mathrm{O}_{2}: \mathrm{C} 61.39, \mathrm{H}$ 3.99, N 16.17; found: C 61.34, H 3.97 N 16.11.

(E)-2-(2-(3-bromobenzylidene)-1-isonicotinoylhydrazinyl)- $N$-(4(pyridin-3-yl)-6-(p-tolyl) pyrimidin-2-yl)acetamide $2_{G}$

IR: 3431.13, 3331.42 (N-H), 3032.39, (C-H aromatic), 2932.50 (C-H), 1671.21 (-CONH), 1539.21(N-H bend), 1412.46, 1441.37, 1461.37 (C=C, aromatic), 1353.33, 1274.26, 1218.37, 1183.49, $1140.44(\mathrm{C}-\mathrm{N})$, 573.76 (mono substituted $\mathrm{Br}$ ). ${ }^{1} \mathrm{H}-\mathrm{NMR}\left(\mathrm{CDCl}_{3}, 400 \mathrm{MHz}\right): 8.57(s, 1 \mathrm{H}$, $\mathrm{CH}$ of pyrimidine ring), 7.12-9.25 ( $m, 16 \mathrm{H}, \mathrm{Ar}-\mathrm{H}) ; 8.36(\mathrm{~s}, 1 \mathrm{H}, \mathrm{CH}) ; 5.23$ $(s, 1 \mathrm{H}, \mathrm{CONH}), 3.85\left(s, 2 \mathrm{H}, \mathrm{CH}_{2}\right) ; 2.34\left(s, 3 \mathrm{H}, \mathrm{CH}_{3}\right) \cdot{ }^{13} \mathrm{C}-\mathrm{NMR}\left(\mathrm{CDCl}_{3}, 100\right.$
Table 2: In vitro antibacterial activity (MIC, $\mu \mathrm{g} / \mathrm{mL}$ ) of the synthesized compounds

\begin{tabular}{lllll}
\hline \multirow{2}{*}{ Code number } & \multicolumn{3}{l}{ MIC $\boldsymbol{\mu g} / \mathbf{m L}$} & \\
\cline { 2 - 5 } & E. coli & P. aeruginosa & S. aureus & S. pyogenes \\
\hline $2_{\mathrm{A}}$ & 200 & 100 & 250 & 100 \\
$2_{\mathrm{B}}$ & 250 & 200 & 250 & 125 \\
$2_{\mathrm{C}}$ & 200 & 100 & 500 & 200 \\
$2_{\mathrm{D}}$ & 100 & 125 & 500 & 250 \\
$2_{\mathrm{E}}$ & 250 & 200 & 250 & 250 \\
$2_{\mathrm{F}}$ & 250 & 500 & 500 & 200 \\
$2_{\mathrm{G}}$ & 500 & 250 & 250 & 125 \\
$2_{\mathrm{H}}$ & 100 & 125 & 200 & 125 \\
$2_{\mathrm{I}}$ & 500 & 100 & 125 & 200 \\
$2_{\mathrm{J}}$ & 125 & 250 & 200 & 125 \\
Ampicillin & 100 & 100 & 250 & 100 \\
Chloramphenicol & 50 & 50 & 50 & 50 \\
Ciprofloxacin & 25 & 25 & 50 & 50 \\
\hline
\end{tabular}

MIC: Minimal inhibitory concentration, E. coli: Escherichia coli (MTCC no. 442), P. aeruginosa: Pseudomonas aeruginosa (MTCC no. 441) S. aureus: Staphylococcus aureus (MTCC no. 96), S. pyogenes: Staphylococcus pyogenes (MTCC no. 443), MTCC: Microbial type culture collection

MHz): 165.18; 163.93 (C=0); 149.99-123.23; $55.34\left(\mathrm{CH}_{2}\right), 21.14\left(\mathrm{CH}_{3}\right)$. MS (m/z): $605.13\left([\mathrm{M}+\mathrm{H}]^{+}\right), 606.12\left[\mathrm{M}^{+}+1\right], 607.13\left[\mathrm{M}^{+}+2\right]$, elemental analysis calculated for $\mathrm{C}_{31} \mathrm{H}_{24} \mathrm{BrN}_{7} \mathrm{O}_{2}$ : C 61.39, H 3.99, N 16.17; found: C 61.40, H 3.98 N 16.19 .

(E)-2-(2-(3-bromobenzylidene)-1-isonicotinoylhydrazinyl)- $\mathrm{N}$-(4(pyridin-4-yl)-6-(p-tolyl) pyrimidin-2-yl)acetamide $2_{H}$ IR: $3423.86(\mathrm{~N}-\mathrm{H}), 3032.19,(\mathrm{C}-\mathrm{H}$ aromatic), 1678.21 (-CONH), $1532.21(\mathrm{~N}-\mathrm{H}$ bend), 1412.46, 1451.37, ( $\mathrm{C}=\mathrm{C}$, aromatic), 1353.33, 1274.26, 1218.37, 1176.23, 1148.21 (C-N), 571.76 (mono substituted Br). ${ }^{1} \mathrm{H}-\mathrm{NMR}\left(\mathrm{CDCl}_{3}, 400 \mathrm{MHz}\right): 8.62(s, 1 \mathrm{H}, \mathrm{CH}$ of pyrimidine ring), 7.59-8.77 (m, 16H, Ar-H); 8.39 ( $s, 1 \mathrm{H}, \mathrm{CH}) ; 5.31(s, 1 \mathrm{H}, \mathrm{CONH}) ; 3.85(s$, $\left.2 \mathrm{H}, \mathrm{CH}_{2}\right) ; 2.34(s, 3 \mathrm{H}, \mathrm{CH} 3) .{ }^{13} \mathrm{C}-\mathrm{NMR}\left(\mathrm{CDCl}_{3}, 100 \mathrm{MHz}\right): 168.25,165.66$; $162.76(\mathrm{C}=0) ; 157.73$ (Pyrimidine); 149.71-106.23 (aromatic ring); $55.43\left(\mathrm{CH}_{2}\right), 21.33\left(\mathrm{CH}_{3}\right)$. MS (m/z): $605.13\left([\mathrm{M}+\mathrm{H}]{ }^{+}\right), 606.11\left[\mathrm{M}^{+}+1\right]$, $607.12\left[\mathrm{M}^{+}+2\right]$, elemental analysis calculated for $\mathrm{C}_{31} \mathrm{H}_{24} \mathrm{BrN}_{7} \mathrm{O}_{2}: \mathrm{C} 61.39$, H 3.99, N 16.17; found: C 61.41, H 3.97 N 16.18 .

(E)-2-(2-(3-bromobenzylidene)-1-isonicotinoylhydrazinyl)- $\mathrm{N}$-(4(3-bromophenyl)-6-(p-tolyl)pyrimidin-2-yl)acetamide 2 IR: 3472.56, $3303.45(\mathrm{~N}-\mathrm{H}), 3199.37,3060.39,3032.38$ (C-H aromatic), 2951.44, 2922.4 (C-H), 1683.22 (-CONH), 1546.21 (N-H bend), 1412.36, $1428.37,1472.37$ (C=C, aromatic), 1353.33, 1274.26, 1218.37, 1183.49, 1140.44, 1189.57 (C-N), 573.76 (mono substituted Br). ${ }^{1} \mathrm{H}-\mathrm{NMR}$ ( $\left.\mathrm{CDCl}_{3}, 400 \mathrm{MHz}\right)$ : 7.42-8.77 (m, 17H, Ar-H); $8.39(\mathrm{~s}, 1 \mathrm{H}, \mathrm{CH}) ; 5.34(s$, $1 \mathrm{H}, \mathrm{CONH}) ; 3.85\left(s, 2 \mathrm{H}, \mathrm{CH}_{2}\right) ; 2.34(s, 3 \mathrm{H}, \mathrm{CH} 3) .{ }^{13} \mathrm{C}-\mathrm{NMR}\left(\mathrm{CDCl}_{3}, 100\right.$ MHz): 168.55, 165.76 (C=0); 160.26, 156.03 (pyrimidine); 149.71107.09 (aromatic ring); $55.43\left(\mathrm{CH}_{2}\right), 21.53\left(\mathrm{CH}_{3}\right)$. MS (m/z): 684.03([M $\left.+\mathrm{H}^{+}\right), 686.03\left[\mathrm{M}^{+}+2\right]$, elemental analysis calculated for $\mathrm{C}_{32} \mathrm{H}_{24} \mathrm{Br}_{2} \mathrm{~N}_{6} \mathrm{O}_{2}$ : C 56.16, H 3.53, N 12.28; found: C 56.24, H 3.55, N 12.24 .

(E)-2-(2-(3-bromobenzylidene)-1-isonicotinoylhydrazinyl)- $N$-(4(3-chlorophenyl)-6-(p-tolyl) pyrimidin-2-yl)acetamide 2 , IR: 3472.56, 3303.45 (N-H), 3199.37, 3060.39, 3032.38 (C-H aromatic), 2951.44, 2922.4 (C-H), 1679.22 (-CONH), 1534.21 (N-H bend), 1412.36, 1428.34, 1471.37 (C=C, aromatic), 1351.33, 1264.26, 1218.37, 1183.49, 1140.44 (C-N), 834.23 (C-Cl) 581.76 (mono substituted Br). ${ }^{1} \mathrm{H}-\mathrm{NMR}$ $\left(\mathrm{CDCl}_{3}, 400 \mathrm{MHz}\right)$ : 7.15-8.77 (m, 17H, Ar-H); $8.39(\mathrm{~s}, 1 \mathrm{H}, \mathrm{CH}) ; 5.34(\mathrm{~s}, 1 \mathrm{H}$, $\mathrm{CONH}) ; 3.85\left(\mathrm{~s}, 2 \mathrm{H}, \mathrm{CH}_{2}\right) ; 2.34(\mathrm{~s}, 3 \mathrm{H}, \mathrm{CH} 3) .{ }^{13} \mathrm{C}-\mathrm{NMR}\left(\mathrm{CDCl}_{3}, 100 \mathrm{MHz}\right)$ : 168.89, $165.67(\mathrm{C}=0) ; 160.61,156.03$ (pyrimidine); 149.71-107.80 (aromatic ring); $55.43\left(\mathrm{CH}_{2}\right), 21.3\left(\mathrm{CH}_{3}\right)$. MS (m/z): $638.08\left([\mathrm{M}+\mathrm{H}]{ }^{+}\right)$, $639.08\left[\mathrm{M}^{+}+1\right], 640.03$, elemental analysis calculated for $\mathrm{C}_{32} \mathrm{H}_{24}$ BrClN $_{6}$ $\mathrm{O}_{2}$ : C 60.06, H 3.78, N 13.13; found: C 60.04, H 3.75, N 13.10 . 
Table 3: In vitro antifungal and antitubercular activity (MIC, $\mu \mathrm{g} / \mathrm{mL}$ ) of compounds

\begin{tabular}{lllll}
\hline \multirow{2}{*}{ Code number } & \multicolumn{2}{l}{ Minimal fungicidal concentration $(\boldsymbol{\mu g} / \mathbf{m L})$} & MIC $\boldsymbol{\mu g} / \mathbf{m L}$ M. tuberculosis $\mathbf{H}_{37} \mathbf{R V}$ \\
\cline { 2 - 4 } & \multicolumn{1}{c}{ C. albicans } & A. iger & A. clavatus & \\
\hline $2_{\mathrm{A}}$ & 500 & 1000 & 1000 & 250 \\
$2_{\mathrm{B}}$ & 1000 & 500 & 1000 & 500 \\
$2_{\mathrm{C}}$ & 1000 & $>1000$ & $>1000$ & 250 \\
$2_{\mathrm{D}}$ & 500 & 1000 & $>1000$ & 500 \\
$2_{\mathrm{E}}$ & 500 & $>1000$ & $>1000$ & 1000 \\
$2_{\mathrm{F}}$ & 1000 & 500 & $>1000$ & 1000 \\
$2_{\mathrm{G}}$ & 1000 & 250 & 1000 & 250 \\
$2_{\mathrm{H}}$ & $>1000$ & 200 & 500 & 250 \\
$2_{\mathrm{I}}$ & $>1000$ & 200 & 250 & 62.5 \\
$2_{\mathrm{J}}$ & $>1000$ & 100 & 500 & - \\
Griseofulvin & 500 & - & 100 & - \\
Nystatin & 100 & - & 100 & 40 \\
Rifampicin & - & - & 0.20 \\
Isoniazid & - & - & \\
\hline
\end{tabular}

MIC: Minimal inhibitory concentration, C. albicans: Candida albicans (MTCC no. 227), A. niger: Aspergillus niger (MTCC no. 282), A. clavatus: Aspergillus clavatus (MTCC no. 1323), M. tuberculosis: Mycobacterium tuberculosis MTCC: Microbial type culture collection

\section{In vitro antimicrobial and antituberculosis activity}

All the newly synthesized compounds were screened for their antimicrobial activity. This activity was determined by the broth micro dilution method according to the National Committee for Clinical Laboratory Standards [29,30]. For antibacterial activity, we used S. aureus microbial type culture collection (MTCC 96) and S. pyogenes (MTCC 443) as Gram-positive, E. coli (MTCC 442) and P. aeruginosa (MTCC 441) as Gram-negative strains using ampicillin, chloramphenicol, and ciprofloxacin as a standard antibacterial drug. Antifungal activity was screened for three different fungal spices C. albicans (MTCC 227), A. niger (MTCC 282), and A. clavatus (MTCC 1323). Griseofulvin and nystatin used as a standard antifungal drug. The strains were procured from Institute of Microbial Technology, Chandigarh.

\section{DISCUSSION}

Pyrimidine derivatives have been very well known in medicinal chemistry for their therapeutic applications. The presence of a pyrimidine base in thymine, cytosine and uracil, which are the essential binding blocks of nucleic acids, DNA and RNA is one possible reason for their activity. The literature indicated that compounds having pyrimidine nucleus possess a broad range of biological activities. Like 5-fluorouracil as anticancer; idoxuridine and trifluridine as antiviral; zidovudine and stavudine as anti-HIV, trimethoprim, sulfamethazine, and sulfadiazine as antibacterial; sulfadoxine as antimalarial and antibacterial; minoxidil and prazosin as antihypertensive; barbiturates, e.g., phenobarbitone as sedative, hypnotics and anticonvulsant; propylthiouracil as antithyroid; thonzylamine as H1-antihistamine; toxoflavin and fervenulin as antibiotics. In this study, pyrimidine clubbed Schiff base of isoniazid have been taken for dual evaluations antimicrobial as well as antituberculosis. Isoniazid itself used as firstline antituberculosis drug. When the Schiff of isoniazid clubbed with pyrimidine moiety the resulted compounds revealed good activities. Compounds $2_{\mathrm{A}-\mathrm{E}}$ with fluorine group and $2_{\mathrm{G}-\mathrm{I}}$ with a methyl group showed good antibacterial activity. Whereas fluorine group containing compounds $2_{\mathrm{A}^{\prime}} 2_{\mathrm{D}^{\prime}}$ and $2_{\mathrm{E}}$ was active toward $C$. albicans. Only one compound 2 , showed good potential toward M. tuberculosis $H_{37} R V$. All the compounds of this series with fluorine and the methyl group showed good potential as an antimicrobial agent and antituberculosis also which open door for future investigation. The results of all compounds against antibacterial activity were displayed in Table 2 . Antifungal and antituberculosis activity results of the all compounds were displayed in Table 3. All synthesized compounds conducted at $250 \mu \mathrm{g} / \mathrm{mL}, 500 \mu \mathrm{g} / \mathrm{mL}$, and $1000 \mu \mathrm{g} / \mathrm{mL}$ for antituberculosis activity.

\section{CONCLUSION}

Most of the synthesized compounds showed good antibacterial activity against Gram-positive as well as Gram-negative bacteria.
Compounds $2_{\mathrm{D}}$ and $2_{\mathrm{H}}$ were equally active against $E$. coli. Compound $2_{\mathrm{A}^{\prime}} 2_{\mathrm{C}^{\prime}}$, and $2_{1}$ showed very good activity toward P. aeruginosa. $2_{\mathrm{A}^{\prime}} 2_{\mathrm{B}^{\prime}}$ $2_{\mathrm{E}}, 2_{\mathrm{G}}, 2_{\mathrm{H}}, 2_{\mathrm{V}}$, and 2 showed excellent activity toward $S$. aureus. Only $2_{\mathrm{A}}$ showed good activity against $S$. pyogenes as compared to standard drug ampicillin. Antifungal activity of compound $2_{A^{\prime}} 2_{D}$, and $2_{E}$ equal against C. albicans compared with standard drug Griseofulvin. Other compounds were moderately sensitive toward A. niger and A. clavatus. All the compounds were active against Mycobacterium tuberculosis $\mathrm{H}_{37} \mathrm{RV}$, but mainly more active compounds were $2_{\mathrm{A}^{\prime}}, 2_{\mathrm{B}^{\prime}}$ $2_{\mathrm{C}^{\prime}}, 2_{\mathrm{D}}, 2_{\mathrm{G}}, 2_{\mathrm{H}}$, and $2_{\mathrm{J}}$. Among of them, compound $2_{\mathrm{J}}$ was highly active moiety as compared to another compound. The final conclusion of this series is that this class of molecules certainly holds great promise toward the pursuit to discover the novel class of antimicrobial and antituberculosis agents.

\section{ACKNOWLEDGMENT}

The authors are thankful to The Head, Department of Chemistry, Veer Narmad South Gujarat University, Surat, for providing necessary facilities to carry out this work. We are grateful to the Director, CIL and SAIF, Panjab University for providing spectral data and the Director, MicroCare Laboratory, Surat, for biological evaluation.

\section{REFERENCES}

1. Cade CE, Dlouhy AC, Medzihradszky KF, Salas-Castillo SP, Ghiladi RA. Isoniazid-resistance conferring mutations in Mycobacterium tuberculosis KatG: Catalase, peroxidase, and INHNADH adduct formation activities. Protein Sci 2010;19(3):458-74.

2. Lourenço MC, De Souza MV, Pinheiro AC, Ferreira ML, Gonçalves RS, Nogueira TC. Evaluation of anti-tubercular activity of nicotinic and isoniazid analogues. Arkivoc 2007;15:181-91.

3. Thomas B, Harindran J. Design, synthesis and evaluation of anti tubercular activity of amino a zetidinones from isoniazid. Int J Phrm Sci Res 2016;7(7):2795-804.

4. Kachroo M, Panda R, Yadav Y. Synthesis and biological activities of some new pyrimidine derivatives from chalcones. Pharm Chem 2014;6(2):352-9.

5. Patel PN, Karia DC. Synthesis and bioactivity evaluation of novel bipyenylthioxo-pyrimidines as potent antimicrobial agent. Pharm Chem 2015;7(10):427-33.

6. Kumaresan S, Chandrasekaran S, Sakthivel KM, Guruvayoorappan C, Enoch IV. Synthesis, characterization and anti-inflammatory activity of some novel pyrimidin-2-amines on carrageenan-induced paw edema in balb/c mice. J Chem Pharm Res 2014;6(10):593-606.

7. Bano T, Kumar N, Dudhe R. Free radical scavenging properties of pyrimidine derivatives. Org Med Chem Lett 2012;2(1):34

8. Mohana KN, Basavapatna N, Kumar P, Mallesha L. Synthesis and biological activity of some pyrimidine derivatives. Drug Invent Today 2013;5(3):216-22

9. Kaur N, Aggarwal AK, Sharma N, Choudhary B. Synthesis and in-vitro 
antimicrobial activity of pyrimidine derivatives. Int J Pharm Sci Drug Res 2012;4(3):199-204.

10. Punkvang A, Saparpakorn P, Hannongbua S, Wolschann P, Pungpo P. Elucidating drug-enzyme interactions and their structural basis for improving the affinity and potency of isoniazid and its derivatives based on computer modeling approaches. Molecules 2010;15(4):2791-813.

11. Sahu S, Siddiqui N. A review on biological importance of pyrimidines in the New Era. Int J Pharm Pharm Sci 2016;8(5):8-21.

12. Kaur R, Kaur P, Sharma S, Singh G, Mehndiratta S, Bedi PM, et al. Anti-cancer pyrimidines in diverse scaffolds: A review of patent literature. Recent Pat Anticancer Drug Discov 2015;10(1):23-71.

13. Phoujdar MS, Aland GR. Molecular docking study on $1 \mathrm{H}-(3,4 \mathrm{~d})$ pyrazolo-pyrimidines as cyclin dependant kinase (cdk2) inhibitors. Int J Curr Pharm Res 2017;9(1):94-100.

14. David VB, Babu VH, Reddy BM. Synthesis of novel 4,6-disubstituted2-amino pyrimidines as antibacterial agents. Int J Pharm Chem Res 2014;3(14):1-6.

15. Patel NB, Patel HR. Design and synthesis of new imidazolinone derivatives as a potential anti fungal agents. J Heterocycl Chem 2011;48:373-80

16. Patel NB, Patel HR. Synthesis and pharmacological studies of 5-ethylpyridin-2-ethanol analogs derivatives. Arkivok 2009;12:302-21.

17. Patel NB, Patel HR. Design and synthesis of 2-(5-ethyl-pyridine-2-yl) ethanol analogs as potential microbial agents. Int J Drug Des Discov 2010;1(1):93-106.

18. Agarwal A, Kumar B, Mehrotra PK, Chauhan PM. 2,4,6-trisubstituted pyrimidine derivatives as pregnancy interceptive agents. Bioorg Med Chem 2005;13(6):1893-9.

19. Sunduru N, Agarwal A, Katiyar SB, Nishi, Goyal N, Gupta S, et al. Synthesis of 2,4,6-trisubstituted pyrimidine and triazine heterocycles as antileishmanial agents. Bioorg Med Chem 2006;14(23):7706-15.

20. Badavath VN, Sinha BN, Jayaprakash V. Design, in-silico docking and predictive ADME properties of novel pyrazoline derivatives with selective human MAO inhibitory activity. Int J Pharm Pharm Sci 2015;7(12):277-82.

21. Rammohan $\mathrm{P}$, Taradas S, Tapas S. Improved microwave-induced synthesis of indolylchalcones. Arch Appl Sci Res 2014;6(3):138-41.

22. Mathew B, Unnikirishnan G, Shafeer VP, Mohammed MC, Femina P. Microwave assisted synthesis, physicochemical properties and antimicrobial activity of benzimidazole chalcones. Pharm Chem 2011;3(6):627-31

23. Revathi R, Sree CR, Jayakumar R, Visagaperumal D, Anbalagan N. Microwave assisted synthesis and biological activity of certain 4-hydroxy chalcones. Pharmacophore 2013;4(2):59-69.

24. Sweta S, Choudhary PC, Intodia K. Microwave irradiation synthesis of various substituted chalcones using various heterogeneous catalysts under solvent-free conditions and their biological study. Chem Sci Trans 2013;2(2):343-8.

25. Karthikeyan E, Mohammed AA, Manogaran E, Kalpana E, Sivaneswari S, Kumar MS, et al. Design, synthesis and biological evaluation of some novel isoniazid cyclocondensed azitidinone. Drug Invent Today 2013;5:100-4.

26. Nalini CN, Arivukkarasi, Devi R. Structure based drug design, synthesis, characterization and biological evaluation of novel isoniazid derivatives. Rasayan J Chem 2011;4(4):868-74.

27. Rattan A, Churchill BI. Antimicrobials in Laboratory Medicine. New Delhi: Livingstone; 2000. p. 85.

28. Anargyros P, Astill DS, Lim IS. Comparison of improved BACTEC and Lowenstein-Jensen media for culture of mycobacteria from clinical specimens. J Clin Microbiol 1990;28(6):1288-91.

29. The National Committee for Clinical Laboratory Standard. Reference Method for Broth Dilution Antifungal Susceptibility Testing of Yeasts Approved Standard M27A. Wayne, PA: NCCLS; 1997.

30. Robert C. Medical Microbiology. $11^{\text {th }}$ ed. Briton: ELBS and E \& S., Livingstone; 1970. p. 89. 